\title{
Evaluation of a trastuzumab-containing treatment regimen for patients with unresectable advanced or recurrent gastric cancer
}

\author{
TSUTOMU NAMIKAWA $^{1}$, ERI MUNEKAGE ${ }^{1}$, MASAYA MUNEKAGE $^{1}$, \\ HIROMICHI MAEDA ${ }^{2}$, TOMOAKI YATABE ${ }^{3}$, HIROYUKI KITAGAWA ${ }^{1}$, KOUICHI SAKAMOTO $^{1}$, \\ MASAYUKI OBATAKE ${ }^{1}$, MICHIYA KOBAYASHI ${ }^{2,4}$ and KAZUHIRO HANAZAKI ${ }^{1}$
}

${ }^{1}$ Department of Surgery, Kochi Medical School, Kochi 783-8505; ${ }^{2}$ Cancer Treatment Center, Kochi Medical School Hospital; Departments of ${ }^{3}$ Anesthesiology and ${ }^{4}$ Human Health and Medical Sciences, Kochi Medical School, Kochi 783-8505, Japan

Received January 21, 2016; Accepted April 21, 2016

DOI: $10.3892 / \mathrm{mco} .2016 .892$

\begin{abstract}
The present study aimed to evaluate the efficacy and safety of trastuzumab plus chemotherapy for patients with unresectable advanced or recurrent gastric cancer. A retrospective analysis of 213 patients with unresectable advanced or recurrent gastric cancer who received systemic chemotherapy,including 15 patients who were also administered trastuzumab, at Kochi Medical School between 2007 and 2013 was performed. The overall survival was compared between patients who received trastuzumab plus chemotherapy and patients who received chemotherapy alone, and the safety and efficacy of the trastuzumab-containing regimen was evaluated. Human epidermal growth factor receptor (HER)2 status was examined in 86 patients, of whom 15 (17.4\%) exhibited strong positive HER 2 expression. The rate of strong positive HER2 expression was significantly higher for intestinal type tumors compared with diffuse type tumors [23.6 (13/55) vs. $6.5 \%(2 / 31) ; \mathrm{P}=0.044]$. The median overall survival of the patients treated with trastuzumab was significantly longer compared with that for patients who were not treated with trastuzumab (22.9 vs. 11.6 months; $\mathrm{P}=0.014$ ). The objective response rate and disease control rate for trastuzumab plus chemotherapy were 46.7 and $86.7 \%$, respectively. Frequently encountered grade 3-4 toxicities included neutropenia $(26.7 \%$; $4 / 15)$, anemia $(13.3 \% ; 2 / 15)$ and fatigue $(13.3 \% ; 2 / 15)$. Trastuzumab plus chemotherapy is effective for patients with HER2-positive advanced or recurrent gastric cancer, and the frequencies of hematological and non-hematological toxicities experienced by patients in the present study indicated that it can be safely administered clinically.
\end{abstract}

Correspondence to: Dr Tsutomu Namikawa, Department of Surgery, Kochi Medical School, Kohasu, Oko-cho, Nankoku, Kochi 783-8505, Japan

E-mail: tsutomun@kochi-u.ac.jp

Key words: gastric cancer, trastuzumab, human epidermal growth factor receptor 2

\section{Introduction}

Gastric cancer is the third most commonly diagnosed cancer type worldwide and one of the leading causes of cancer-associated mortality (1). In patients with recurrent, metastatic, or advanced gastric cancer, chemotherapy can prolong survival and improve quality of life compared with the best supportive care.

Human epidermal growth factor receptor (HER)2 is a member of a family of receptors that is associated with tumor cell proliferation, apoptosis, adhesion, migration and differentiation. It is overexpressed in $15-20 \%$ of patients with primary gastric and gastroesophageal junction cancers $(2,3)$. Trastuzumab, a monoclonal antibody that targets HER2, induces antibody-dependent cellular cytotoxicity and inhibits HER2-mediated signaling by binding to the extracellular domain of HER2 (4). The trastuzumab for gastric cancer(ToGA) trial recently validated the additive effects of trastuzumab for HER2-positive, unresectable advanced or recurrent gastric cancer. This trial revealed a significant increase in the overall survival and progression-free survival when trastuzumab was used in combination with chemotherapy, thus supporting the use of trastuzumab in individualized, biomarker-based treatment (5). Additionally, it has been shown that second-line and later lines of chemotherapy can significantly improve patient outcomes, and that the additive effect of molecular targeted drug therapy is promising $(4,6-10)$.

In the present study, the clinicopathological features and outcomes of patients with HER2-positive gastric cancer who were treated with trastuzumab and cytotoxic anti-cancer agents were analyzed.

\section{Patients and methods}

Study design. The present study performed a retrospective review of patients with unresectable advanced or recurrent gastric cancer who were treated with systemic chemotherapy.

Patients. Patients with unresectable advanced or recurrent gastric cancer who were treated with chemotherapy at Kochi Medical School (Kochi, Japan) between January 2007 and December 2013 were identified from a medical information 
database. Gastric cancer diagnoses were determined by esophagogastroduodenoscopy, biopsy specimen analysis, computed tomography, magnetic resonance imaging, ultrasonography of the abdomen and positron emission tomography.

HER2 testing and treatment. In 86/213 patients, HER 2 status was examined by immunohistochemical (IHC) staining using tumor specimens obtained by endoscopic biopsy or obtained from resected primary lesions, according to the IHC scoring criteria for gastric cacner (11). If the IHC score was $2+$, fluorescence in-situ hybridization (FISH) analyses were performed using DNA probes (PathVysion HER2 DNA Probe kit; Abbott, Tokyo, Japan). The histological type for each tumor was categorized as intestinal type (well differentiated, moderately differentiated and papillary adenocarcinoma) or diffuse type (poorly differentiated, mucinous adenocarcinoma and signet ring cell carcinoma), according to Lauren (12) classification. If a patient tumor sample scored $3+$ on IHC or scored $2+$ on IHC and was FISH positive, the patient was treated with trastuzumab plus chemotherapy.

In the trastuzumab plus chemotherapy regimen, chemotherapy was administered every 3 weeks until disease progression, unacceptable toxicity, or withdrawal of consent. Capecitabine $\left(1,000 \mathrm{mg} / \mathrm{m}^{2}\right)$ was administered orally twice a day for 14 days, and this was followed by a 1 week rest period. Cisplatin $\left(80 \mathrm{mg} / \mathrm{m}^{2}\right)$ was administered by intravenous infusion on day 1 . Trastuzumab $(8 \mathrm{mg} / \mathrm{kg})$ was administered by intravenous infusion on day 1 of the first cycle of chemotherapy, followed by a dose of $6 \mathrm{mg} / \mathrm{kg}$ every 3 weeks.

Safety and efficacy evaluation. Toxicity was evaluated according to National Cancer Institute Common Terminology Criteria for Adverse Events version 4.0. The clinical tumor response was assessed following two or three courses of chemotherapy, according to criteria in the Response Evaluation Criteria In Solid Tumors (RECIST) guideline version 1.1 (13). Responses were defined as complete response, partial response, stable disease or progressive disease. The overall survival time was defined as the interval between the date of chemotherapy initiation and the date of mortality or last contact. Surviving patients were censored at the last follow-up date.

Statistical analysis. Differences between the mean values for the two groups of patients were assessed for significance using the Mann-Whitney $U$ test for continuous variables and the Pearson's $\chi^{2}$ test for categorical variables. The Kaplan-Meier method was used to generate cumulative survival rates and these were compared using the log-rank test to evaluate statistically significant differences. Statistical analyses were performed using SPSS for Windows, version 13.0 (SPSS Inc, Chicago, IL, USA).

\section{Results}

Patients characteristics. A total of 213 patients with unresectable advanced or recurrent gastric cancer were identified. Patient clinical characteristics are summarized in Table I. A total of 15 patients ( 13 men/2 women) received trastuzumab plus chemotherapy. The median age of this group was 66-years-old (range, 49-82 years). Of these 15 patients, 13 had an intestinal type tumor and 2 had a diffuse type tumor, 12 were classified as having metastatic cancer at the time of diagnosis, and 3 were classified as having recurrent cancer following curative resection of gastric cancer.

The prevalence of intestinal type tumors was significantly higher in the trastuzumab plus chemotherapy group compared with in the chemotherapy alone group (86.7 vs. $33.3 \%$; $\mathrm{P}<0.001)$. No significant differences in age, sex or disease status were clear at the time of diagnosis, and metastatic site between the trastuzumab plus chemotherapy and chemotherapy alone groups. Of the 15 patients in the trastuzumab plus chemotherapy group, 14 exhibited tumor specimens that scored IHC $3+$ and the other exhibited a tumor specimen that scored IHC 2+ and was FISH positive.

Clinicopathological characteristics of HER2-positive patients. HER 2 testing of tumor specimens was undertaken for a total of 86 patients. The IHC scores of $0,1+, 2+$ and $3+$ were revealed for $37,21,14$ and 14 of these patients, respectively. Of those with an IHC score of $2+$, one was FISH positive, and the rate of the strong positive HER2 expression was $17.4 \%(15 / 86)$. Intestinal type tumors had a significantly higher rate of strong positive HER2 expression compared with diffuse type tumors [23.6\% (13/55) vs. $6.5 \%(2 / 31) ; \mathrm{P}=0.044]$.

Safety of the trastuzumab plus chemotherapy regimen. The main hematological and non-hematological adverse events experienced by patients in the trastuzumab plus chemotherapy group during all cycles of treatment are shown in Table II. Among the hematological adverse events, the proportions of patients who had grade 3-4 neutropenia and anemia were 26.7 and $13.3 \%$, respectively. Creatinine levels were elevated in $40 \%$ of patients. The most common non-hematological adverse event was fatigue (all grades, 46.7\%; grades $3-4,13.3 \%$ ). With the exception of fatigue, no grade 3 or 4 non-hematological adverse events occurred. Only 1 patient (6.7\%) exhibited a grade 2 infusion-associated reaction, which was alleviated by symptomatic therapy, and it was possible to restart trastuzumab administration for this patient with diclofenac sodium premedication and an adjusted trastuzumab dosage rate. No patients suffered from heart failure and no treatment-associated mortalities occurred.

Tumor response and patient survival. Objective tumor responses of patients treated with trastuzumab plus chemotherapy, based on RECIST criteria, are shown in Table III. The overall objective response rate and disease control rate were 46.7 (7/15) and $86.7 \%$ (13/15). Of these patients, 1 had a complete response, 6 had a partial response, 6 had stable disease and 2 had progressive disease. For the trastuzumab plus chemotherapy group, the median observation period at the time of the analysis was 28.5 months, and the median duration of treatment with trastuzumab was 13 months.

The median overall survival time for the trastuzumab plus chemotherapy group was 22.9 months, which was significantly longer compared with that for the chemotherapy alone group (11.6 months; $\mathrm{P}=0.014$; Fig. 1). Second-line therapy after disease progression was administered to all patients in the 
Table I. Patient characteristics.

\begin{tabular}{|c|c|c|c|}
\hline \multirow[b]{2}{*}{ Characteristic } & \multicolumn{2}{|c|}{ No. patients } & \multirow[b]{2}{*}{ P-value } \\
\hline & Trastuzumab plus chemotherapy $(\mathrm{n}=15)$ & Chemotherapy alone $(n=198)$ & \\
\hline Median age, years (range) & $66(49-82)$ & $70(19-89)$ & 0.574 \\
\hline \multicolumn{4}{|l|}{ Gender (\%) } \\
\hline Male & 13 & 124 & \multirow{2}{*}{0.111} \\
\hline Female & 2 & 74 & \\
\hline \multicolumn{4}{|l|}{ Histology } \\
\hline Intestinal type & 13 & 66 & \multirow{2}{*}{$<0.001$} \\
\hline Diffuse type & 2 & 132 & \\
\hline \multicolumn{4}{|l|}{ Disease status } \\
\hline Initially metastatic & 12 & 140 & \multirow{2}{*}{0.722} \\
\hline Recurrence after curative resection & 3 & 54 & \\
\hline \multicolumn{4}{|l|}{ Metastatic site } \\
\hline Liver & 9 & 55 & \multirow{5}{*}{0.218} \\
\hline Peritoneum & 3 & 96 & \\
\hline Lymph node & 3 & 37 & \\
\hline Lung & 1 & 8 & \\
\hline Bone & 0 & 11 & \\
\hline
\end{tabular}

Table II. Adverse events experienced by the 15 patients in the trastuzumab plus chemotherapy group during all cycles of treatment.

\begin{tabular}{|c|c|c|c|c|c|}
\hline \multirow[b]{2}{*}{ Adverse event } & \multicolumn{5}{|c|}{ Number of patients (\%) } \\
\hline & All grades & Grade 1 & Grade 2 & Grade 3 & Grade 4 \\
\hline \multicolumn{6}{|l|}{ Non-hematological } \\
\hline Fatigue & $7(46.7)$ & $2(13.3)$ & $3(20.0)$ & $2(13.3)$ & 0 \\
\hline Anorexia & $5(33.3)$ & $3(20.0)$ & $2(13.3)$ & 0 & 0 \\
\hline Nausea & $5(33.3)$ & $3(20.0)$ & $2(13.3)$ & 0 & 0 \\
\hline Diarrhea & $2(13.3)$ & $1(6.7)$ & $1(6.7)$ & 0 & 0 \\
\hline Constipation & $1(6.7)$ & $1(6.7)$ & 0 & 0 & 0 \\
\hline Stomatitis & $2(13.3)$ & $1(6.7)$ & $1(6.7)$ & 0 & 0 \\
\hline Hand-foot syndrome & $4(26.7)$ & $3(20.0)$ & $1(6.7)$ & 0 & 0 \\
\hline Infusion-related reaction & $1(6.7)$ & 0 & $1(6.7)$ & 0 & 0 \\
\hline \multicolumn{6}{|l|}{ Hematological } \\
\hline Leukopenia & $10(66.7)$ & $3(20.0)$ & $4(26.7)$ & $3(20.0)$ & \\
\hline Neutropenia & $11(73.3)$ & $3(20.0)$ & $4(26.7)$ & $3(20.0)$ & $1(6.7)$ \\
\hline Thrombocytopenia & $5(33.3)$ & $5(33.3)$ & 0 & 0 & 0 \\
\hline Anemia & $8(53.3)$ & $3(20.0)$ & $3(20.0)$ & $2(13.3)$ & \\
\hline Creatinine elevation & $6(40.0)$ & $5(33.3)$ & $1(6.7)$ & 0 & 0 \\
\hline
\end{tabular}

trastuzumab plus chemotherapy group. Trastuzumab administration during second and later lines of chemotherapy was continued beyond progression for 7 patients (46.7\%).

\section{Discussion}

The present results revealed that trastuzumab plus chemotherapy is an effective and feasible treatment option for patients with HER2-positive metastatic gastric cancer. Trastuzumab is a potent targeted drug that exhibits cytostatic action; it brings about antiproliferative effects on tumor cells via inhibition of HER2 signaling and antibody-dependent cellular cytotoxicity. The ToGA trial revealed that patients with HER2-positive advanced gastric cancer, who were treated trastuzumab in combination with cisplatin plus capecitabine or fluorouracil, had a median overall survival of 13.8 months, compared with 11.1 months for patients in the control group who received chemotherapy alone. Furthermore, it demonstrated that 
Table III. Objective tumor responses for 15 patients treated with trastuzumab plus chemotherapy, based on Response Evaluation Criteria In Solid Tumors criteria.

\begin{tabular}{lc}
\hline Best overall response & Number of patients (\%) \\
\hline Complete response & $1(6.7)$ \\
Partial response & $6(40.0)$ \\
Stable disease & $6(40.0)$ \\
Progressive disease & $2(13.3)$ \\
Objective response rate & $46.7 \%$ \\
Disease control rate & $86.7 \%$ \\
\hline
\end{tabular}

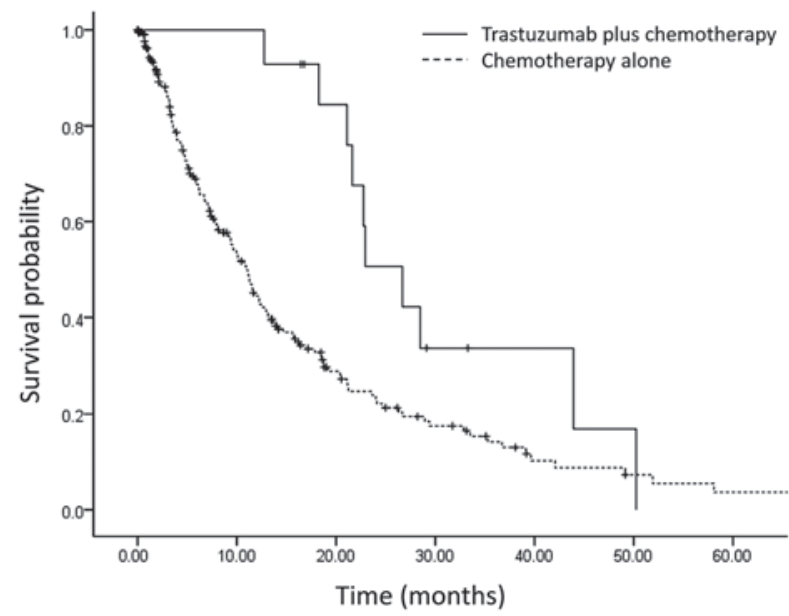

Figure 1. Kaplan-Meier estimates of overall survival in patients treated with chemotherapy, with or without trastuzumab. The median survival was 22.9 months in the trastuzumab plus chemotherapy group, compared with 11.1 months in the group treated with chemotherapy alone. There was a significant difference in survival between the two groups $(\mathrm{P}=0.014$; stratified log-rank test).

patients with higher levels of HER2 expression had a median overall survival of 16.0 months (4). Following publication of the results of the ToGA study, trastuzumab was recommended for use with chemotherapy as a first-line treatment for patients with HER2-positive unresectable advanced or recurrent gastric cancer. The present results are similar to those of the ToGA study. The median overall survival time for patients treated with trastuzumab was significantly longer compared with patients in the chemotherapy alone group (22.9 vs. 11.6 months).

High efficacy of trastuzumab has been confirmed prior to and following chemotherapy in patients with HER2-positive breast cancer, and expanded indications in gastric cancer are anticipated $(14,15)$. Previous studies that have assessed chemotherapy plus trastuzumab treatment regimens for patients with HER2-positive gastric cancer have revealed good tolerance and high efficacy with cytotoxic chemotherapy including S-1 plus cisplatin, capecitabine plus oxaliplatin (or docetaxel), and cisplatin plus $\mathrm{S}-1$, however, not capecitabine plus cisplatin (16-21). Therefore, trastuzumab with cytotoxic chemotherapy is considered a standard treatment regimen for patients with HER2-positive gastric cancer. However, the potential benefits of using trastuzumab in combination with second-line chemotherapy following disease progression and following the use of trastuzumab in combination with first-line therapy remain to be determined.

Other previous studies have shown that second-line therapy can significantly improve survival of patients with unresectable advanced or recurrent gastric cancer who are treated with first-line chemotherapy that includes fluoropyrimidines and platinum (5-7). Furthermore, ramucirumab, a human immunoglobulin G1 monoclonal antibody vascular endothelial growth factor receptor 2 antagonist, with or without paclitaxel, significantly increases the overall survival compared with the placebo in patients with unresectable advanced or recurrent gastric cancer that has progressed following first-line chemotherapy $(8,9)$. Trastuzumab therapy may contribute to improved treatment outcomes following disease progression; however, further investigations, including prospective randomized controlled trials, are required to confirm this.

In the present study, certain patients in the trastuzumab plus chemotherapy group developed neutropenia and thrombocytopenia, likely induced by chemotherapy. These were mitigated by reducing the dose of capecitabine or cisplatin. In addition, no cases of cardiac failure or mortality due to cardiac toxicity were observed. Similarly, previous reports have shown that adding trastuzumab to chemotherapy does not increase the toxic effects associated with standard fluoropyrimidine and platinum chemotherapy (4,16-19). The toxicity profile of trastuzumab plus chemotherapy has been shown to be comparable to that for chemotherapy alone in phase II and III trials $(4,20,21)$.

In the present study, the rate of strong positive HER2 expression in patients with gastric cancer was $17.4 \%$, which is consistent with results from previous studies $(3,4,22)$. Gastric cancer differs from breast cancer in that gastric cancer tumors are highly heterogeneous, plus HER2 expression patterns are different in gastric cancer compared with breast cancer. These differences present a range of challenges for HER 2 testing $(22,23)$. It is critical to optimize factors that affect the accuracy of HER2 testing, including the amount of time between specimen extraction and specimen fixation, and the concentration of fixing agent. In the present study and previous research, HER2 overexpression was more common in intestinal type tumors, which are generally associated with a better prognosis compared with tumors with diffuse histology (24). While overexpression of HER2 is a factor that is associated with poor prognosis in patients with breast cancer, its association with prognosis in patients with gastric cancer is widely debated (25). For this reason, improvements in the accuracy of HER2 testing and further research on this topic are required $(26,27)$. Nonetheless, the survival advantage provided by trastuzumab plus chemotherapy in patients with HER2-positive gastric cancer has been confirmed, with data showing that this regimen is efficacious and safe.

The present study does have certain limitations. Firstly, it included the errors and biases inherent in a retrospective, single-center study, and potential confounding factors may not have been counterbalanced completely. Secondly, pathological characteristics differed between HER2-positive and HER2-negative tumor types, as HER2 positivity was significantly associated with histological grade. Thirdly, a variety of chemotherapy regimens were included in the present study, 
which may have contributed to selection bias. Further studies with adequate statistical power and a larger number of patient subgroups are required to reliably and accurately assess the efficacy of trastuzumab continuation beyond disease progression after first-line therapy for advanced gastric cancer.

In conclusion, although this was not a prospective study, the present results suggested that trastuzumab can be safely administered in combination with chemotherapy in clinical practice by managing adverse events, and that trastuzumab may extend survival when used in combination with second and later lines of chemotherapy in patients with HER2-positive unresectable advanced or recurrent gastric cancer. A well-designed prospective study is required to confirm the utility of trastuzumab continuation beyond disease progression.

\section{References}

1. Siegel RL, Miller KD and Jemal A: Cancer statistics, 2015. CA Cancer J Clin 65: 5-29, 2015

2. Begnami MD, Fukuda E, Fregnani JH, Nonogaki S, Montagnini AL, da Costa WL Jr and Soares FA: Prognostic implications of altered human epidermal growth factor receptors (HERs) in gastric carcinomas: HER2 and HER3 are predictors of poor outcome. J Clin Oncol 29: 3030-3036, 2011.

3. Kurokawa Y, Matsuura N, Kimura Y, Adachi S, Fujita J, Imamura H, Kobayashi K, Yokoyama Y, Shaker MN, Takiguchi S, et al: Multicenter large-scale study of prognostic impact of HER2 expression in patients with resectable gastric cancer. Gastric Cancer 18: 691-697, 2015.

4. Kang JH, Lee SI, Lim do H, Park KW, Oh SY, Kwon HC, Hwang IG, Lee SC, Nam E, Shin DB, et al: Salvage chemotherapy for pretreated gastric cancer: A randomized phase III trial comparing chemotherapy plus best supportive care with best supportive care alone. J Clin Oncol 30: 1513-1538, 2012.

5. Bang YJ, Van Cutsem E, Feyereislova A, Chung HC, Shen L, Sawaki A, Lordick F, Ohtsu A, Omuro Y, Satoh T, et al: Trastuzumab in combination with chemotherapy versus chemotherapy alone for treatment of HER2-positive advanced gastric or gastro-oesophageal junction cancer (ToGA): A phase 3, open-label, randomised controlled trial. Lancet 376: 687-697, 2010.

6. Thuss-Patience PC, Kretzschmar A, Bichev D, Deist T, Hinke A, Breithaupt K, Dogan Y, Gebauer B, Schumacher G and Reichardt P: Survival advantage for irinotecan versus best supportive care as second-line chemotherapy in gastric cancer-a randomised phase III study of the Arbeitsgemeinschaft Internistische Onkologie (AIO). Eur J Cancer 47: 2306-2314, 2011.

7. Hironaka S, Ueda S, Yasui H, Nishina T, Tsuda M, Tsumura T, Sugimoto N, Shimodaira H, Tokunaga S, Moriwaki T, et al Randomized, open-label, phase III study comparing irinotecan with paclitaxel in patients with advanced gastric cancer without severe peritoneal metastasis after failure of prior combination chemotherapy using fluoropyrimidine plus platinum: WJOG 4007 trial. J Clin Oncol 31: 4438-4444, 2013.

8. Wilke H, Muro K, Van Cutsem E, Oh SC, Bodoky G, Shimada Y, Hironaka S, Sugimoto N, Lipatov O, Kim TY, et al: Ramucirumab plus paclitaxel versus placebo plus paclitaxel in patients with previously treated advanced gastric or gastro-oesophageal junction adenocarcinoma (RAINBOW): A double-blind, randomised phase 3 trial. Lancet Oncol 15: 1224-1235, 2014.

9. Fuchs CS, Tomasek J, Yong CJ, Dumitru F, Passalacqua R, Goswami C, Safran H, dos Santos LV, Aprile G, Ferry DR, et al: Ramucirumab monotherapy for previously treated advanced gastric or gastro-oesophageal junction adenocarcinoma (REGARD): An international, randomised, multicentre, placebo-controlled, phase 3 trial. Lancet 383: 31-39, 2014.

10. Chrom P, Stec R and Szczylik C: Second-line treatment of advanced gastric cancer: Current options and future perspectives. Anticancer Res 35: 4575-4583, 2015.

11. Hofmann M, Stoss O, Shi D, Büttner R, van de Vijver M, Kim W, Ochiai A, Rüschoff J and Henkel T: Assessment of a HER2 scoring system for gastric cancer: results from a validation study. Histopathology 52: 797-805, 2008
12. Lauren P: The two histological main types of gastric carcinoma: Diffuse and so-called intestinal-type carcinoma. An attempt at a histo-clinical classification. Acta Pathol Microbiol Scand 64: 31-49, 1965

13. Eisenhauer EA, Therasse P, Bogaerts J, Schwartz LH, Sargent D, Ford R, Dancey J, Arbuck S, Gwyther S, Mooney M, et al: New response evaluation criteria in solid tumours: revised RECIST guideline (version 1.1). Eur J Cancer 45: 228-247, 2009.

14. Gomez-Martín C, Lopez-Rios F, Aparicio J, Barriuso J, García-Carbonero R, Pazo R, Rivera F, Salgado M, Salud A, Vázquez-Sequeiros E and Lordick F: A critical review of HER2-positive gastric cancer evaluation and treatment: From trastuzumab, and beyond. Cancer Lett 351: 30-40, 2014.

15. Boku N: HER2-positive gastric cancer. Gastric Cancer 17: 1-12, 2014.

16. Ryu MH, Yoo C, Kim JG, Ryoo BY, Park YS, Park SR, Han HS, Chung IJ, Song EK, Lee KH, et al: Multicenter phase II study of trastuzumab in combination with capecitabine and oxaliplatin for advanced gastric cancer. Eur J Cancer 51: 482-488, 2015.

17. Mitsui Y, Sato Y, Miyamoto H, Fujino Y, Takaoka T, Miyoshi J, Kagawa M, Ohnuma H, Hirakawa M, Kubo T, et al: Trastuzumab in combination with docetaxel/cisplatin/S-1 (DCS) for patients with HER2-positive metastatic gastric cancer: Feasibility and preliminary efficacy. Cancer Chemother Pharmacol 76: 375-382, 2015.

18. Kang YK, Rha SY, Tassone P, Barriuso J, Yu R, Szado T, Garg A and Bang YJ: A phase IIa dose-finding and safety study of first-line pertuzumab in combination with trastuzumab, capecitabine and cisplatin in patients with HER2-positive advanced gastric cancer. Br J Cancer 111: 660-666, 2014.

19. Kurokawa Y, Sugimoto N, Miwa H, Tsuda M, Nishina S, Okuda H, Imamura H, Gamoh M, Sakai D, Shimokawa T, et al: Phase II study of trastuzumab in combination with S-1 plus cisplatin in HER2-positive gastric cancer (HERBIS-1). Br J Cancer 110: 1163-1168, 2014.

20. Chua C, Tan IB, Yamada Y, Rha SY, Yong WP, Ong WS, Tham CK, Ng M, Tai DW, Iwasa S, et al: Phase II study of trastuzumab in combination with S-1 and cisplatin in the first-line treatment of human epidermal growth factor receptor HER 2-positive advanced gastric cancer. Cancer Chemother Pharmacol 76: 397-408, 2015.

21. Honma Y, Shimada Y, Takashima A, Iwasa S, Kato K, Hamaguchi T, Yamada Y, Taniguchi H, Sekine S and Kushima R: Efficacy of S-1 plus cisplatin combination chemotherapy in patients with HER2-positive advanced gastric cancer. Int J Clin Oncol 19: 863-870, 2014.

22. Van Cutsem E, Bang YJ, Feng-Yi F, Xu JM, Lee KW, Jiao SC, Chong JL, López-Sanchez RI, Price T, Gladkov O, et al: HER2 screening data from ToGA: Targeting HER2 in gastric and gastroesophageal junction cancer. Gastric Cancer 18: 476-484, 2015.

23. Ock CY, Lee KW, Kim JW, Kim JS, Kim TY, Lee KH, Han SW, Im SA, Kim TY, Kim WH, et al: Optimal patient selection for trastuzumab treatment in HER2-positive advanced gastric cancer. Clin Cancer Res 21: 2520-2529, 2015.

24. Nashimoto A, Akazawa K, Isobe Y, Miyashiro I, Katai H, Kodera Y, Tsujitani S, Seto Y, Furukawa H, Oda I, et al: Gastric cancer treated in 2002 in Japan: 2009 annual report of the JGCA nationwide registry. Gastric Cancer 16: 1-27, 2013.

25. Qiu MZ, Li Q, Wang ZQ, Liu TS, Liu Q, Wei XL, Jin Y, Wang DS, Ren C, Bai L, et al: HER2-positive patients receiving trastuzumab treatment have a comparable prognosis with HER2-negative advanced gastric cancer patients: A prospective cohort observation. Int J Cancer 134: 2468-2477, 2014.

26. Shitara K, Yatabe Y, Matsuo K, Sugano M, Kondo C, Takahari D, Ura T, Tajika M, Ito S and Muro K: Prognosis of patients with advanced gastric cancer by HER2 status and trastuzumab treatment. Gastric Cancer 16: 261-267, 2013.

27. Namikawa T, Shiga M, Ichikawa K, Kitagawa H, Kobayashi M and Hanazaki K: Metachronous liver and bone metastasis from small early gastric carcinoma without lymph node involvement: A case report. Mol Clin Oncol 1: 249-252, 2013. 